\title{
Klein-Gordon Oscillator Under the Effects of Violation of the Lorentz Symmetry
}

\author{
Faizuddin Ahmed ${ }^{1}$ \\ National Academy Gauripur, Assam, 783331, India
}

\begin{abstract}
In this work, we investigate the behaviour of relativistic quantum oscillator under the effects of Lorentz symmetry violation determined by a tensor $\left(K_{F}\right)_{\mu \nu \alpha \beta}$ out of the Standard Model Extension. We analyze this relativistic system under an inverse radial electric field and a constant magnetic field induced by Lorentz symmetry violation. We see that the presence of Lorentz symmetry breaking terms modified the energy spectrum of the system, and a quantum effect arise due to the dependence of the linear charge density on the quantum numbers of the system.
\end{abstract}

Keywords: Lorentz symmetry violation, Relativistic wave-equations: bound states solutions, scalar potential, electric \& magnetic field, biconfluent Heun's function .

PACS Number(s): 03.65.Pm, 11.30.Cp, 11.30.Qc

\section{Introduction}

The relativistic quantum dynamics of scalar particle by solving the KGoscillator in various space-times background has been investigated by many authors $[1,2,3,4,5,6,7,8,9,10,11]$ in the relativistic quantum mechanics. The Klein-Gordon oscillator [12] was inspired by the Dirac oscillator [13] applied for spin- $\frac{1}{2}$ particle. In this work, we study the relativistic quantum

\footnotetext{
${ }^{1}$ faizuddinahmed15@gmail.com ; faiz4U.enter@rediffmail.com
} 
oscillator in under Lorentz symmetry breaking effects defined by a tensor $\left(K_{F}\right)_{\mu \nu \alpha \beta}$ out of the Standard Model Extension $[14,15,16,17,18,19,20$, $21,22,23]$. We consider the effects of a Coulomb-type potential induced by the Lorentz symmetry violation, and analyze the behaviour of a relativistic quantum oscillator by solving the Klein-Gordon oscillator. For that, a Coulomb-like radial electric field and a uniform magnetic field is induced by the Lorentz symmetry violation in the quantum system, and obtain the solution of the bound state. We show a quantum effect due to the dependence of the magnetic field on the quantum numbers of the system, and the energy eigenvalues and the wave function modified due to the presence of Lorentz symmetry breaking terms that governs the Lorentz symmetry violation out of the Standard Model Extension.

The gauge sector of the Standard Model Extension possess two violating terms that modifies the transport properties of space-time since these terms break the Lorentz symmetry. These two terms are called the CPT-odd sector $[14,15]$ and the CPT-even sector $[24,25]$. The KG-equation under the effects of Lorentz symmetry violation $[14,15,26,27,28,29,30,31,32,33$, 34, 35, 36] is given by

$$
-\frac{\partial^{2} \Psi}{\partial t^{2}}+\vec{\nabla}^{2} \Psi+\frac{\alpha}{4}\left(K_{F}\right)_{\mu \nu \alpha \beta} F^{\mu \nu}(x) F^{\alpha \beta}(x) \Psi=M^{2} \Psi,
$$

where $\alpha$ is a constant, $F_{\mu \nu}(x)=\partial_{\mu} A_{\nu}-\partial_{\nu} A_{\mu}$ is the electromagnetic field tensor, $\left(K_{F}\right)_{\mu \nu \alpha \beta}$ corresponds to a tensor that governs the Lorentz symmetry violation out of the Standard Model Extension and $M$ is the rest mass of the particle.

The structure of this paper is as follows: in section II, we introduce the Lorentz symmetry breaking effects defined by a tensor $\left(K_{F}\right)_{\mu \nu \alpha \beta}$ that governs the Lorentz symmetry violation out of the Standard Model Extension. Then, we analyse the behaviour of the relativistic quantum oscillator by solving the KG-oscillator under the effects of Lorentz symmetry breaking and obtain the bound states solution; in section III, we present our conclusions. 


\section{Relativistic Quantum Oscillator Under the Lorentz Symmetry Breaking Effects}

We consider the Minkowski flat space-time

$$
d s^{2}=-d t^{2}+d r^{2}+r^{2} d \phi^{2}+d z^{2}
$$

where the ranges of the cylindrical coordinates are $-\infty<(t, z)<\infty, r \geq 0$ and $0 \leq \phi \leq 2 \pi$.

For the geometry (2), the KG-equation under the effects of the Lorentz symmetry violation using (1) becomes

$$
\begin{aligned}
& {\left[-\frac{\partial^{2}}{\partial t^{2}}+\frac{1}{r} \frac{\partial}{\partial r}\left(r \frac{\partial}{\partial r}\right)+\frac{1}{r^{2}} \frac{\partial^{2}}{\partial \phi^{2}}+\frac{\partial^{2}}{\partial z^{2}}\right] \Psi+\frac{\alpha}{4}\left(K_{F}\right)_{\mu \nu \alpha \beta} F^{\mu \nu}(x) F^{\alpha \beta}(x) \Psi} \\
& =M^{2} \Psi
\end{aligned}
$$

One of the properties of the tensor $\left(K_{F}\right)_{\mu \nu \alpha \beta}$ is that it can be written in terms of $3 \times 3$ matrices

$$
\begin{aligned}
& \left(\kappa_{D E}\right)_{j k}=-2\left(K_{F}\right)_{0 j 0 k} \\
& \left(\kappa_{H B}\right)_{j k}=\frac{1}{2} \epsilon_{j p q} \epsilon_{k l m}\left(K_{F}\right)^{p q l m} \\
& \left(\kappa_{D B}\right)_{j k}=-\left(\kappa_{H E}\right)_{k j}=\epsilon_{k p q}\left(K_{F}\right)^{0 j p q}
\end{aligned}
$$

Note that the matrices $\left(\kappa_{D E}\right)_{j k}$ and $\left(\kappa_{H B}\right)_{j k}$ are symmetric, and represent the parity-even sector of the tensor field $\left(K_{F}\right)_{\mu \nu \alpha \beta}$. On the other hand, the matrices $\left(\kappa_{D B}\right)_{j k}$ and $\left(\kappa_{H E}\right)_{k j}$ have no symmetry, and represent the parityodd sector of the tensor field $\left(K_{F}\right)_{\mu \nu \alpha \beta}$. In this way, we can rewrite (3) in the form :

$$
\begin{aligned}
& {\left[-\frac{\partial^{2}}{\partial t^{2}}+\frac{\partial^{2}}{\partial r^{2}}+\frac{1}{r} \frac{\partial}{\partial r}+\frac{1}{r^{2}} \frac{\partial^{2}}{\partial \phi^{2}}+\frac{\partial^{2}}{\partial z^{2}}\right] \Psi } \\
+ & {\left[-\frac{\alpha}{2}\left(\kappa_{D E}\right)_{i j} E^{i} E^{j}+\frac{\alpha}{2}\left(\kappa_{H B}\right)_{j k} B^{i} B^{j}-\alpha\left(\kappa_{D B}\right)_{j k} E^{i} B^{j}\right] \Psi=M^{2} \Psi . }
\end{aligned}
$$

Let us consider a possible scenario of the Lorentz symmetry violation determined by the non-null terms $\left(\kappa_{D E}\right)_{11}=$ const, $\left(\kappa_{H B}\right)_{33}=$ const and 
$\left(\kappa_{D B}\right)_{13}=$ const, and the field configuration is given by $[29,30]$ :

$$
\vec{B}=B_{0} \hat{z} \quad, \quad \vec{E}=\frac{\lambda}{r} \hat{r}
$$

where $B_{0}>0, \hat{z}$ is a unit vector in the $z$-direction, $\lambda$ is a constant associated with a linear distribution of electric charge along the axial direction, and $\hat{r}$ is the unit vector in the radial direction.

To include oscillator with the Klein-Gordon field, we change the following momentum operator $[1,2,3,4,5,6,7,8,9,10,11]$ :

$$
\vec{p} \rightarrow \vec{p}+i M \omega \vec{r}
$$

where $\omega$ is the oscillator frequency and $\vec{r}=r \hat{r}$ with $r$ being the distance from the particle to the axis. So we can replace $\vec{p}^{2} \rightarrow(\vec{p}+i M \omega \vec{r})(\vec{p}-i M \omega \vec{r})$.

Therefore, using the Lorentz symmetry violation term defined above with the field configuration (6) and finally using (7), equation (5) becomes

$$
\begin{aligned}
& {\left[-\frac{\partial^{2}}{\partial t^{2}}+\frac{1}{r}\left(\frac{\partial}{\partial r}+M \omega r\right)\left(r \frac{\partial}{\partial r}-M \omega r^{2}\right)+\frac{1}{r^{2}} \frac{\partial^{2}}{\partial \phi^{2}}+\frac{\partial^{2}}{\partial z^{2}}\right] \Psi} \\
& +\left[-\frac{\alpha}{2}\left(\kappa_{D E}\right)_{11} \frac{\lambda^{2}}{r^{2}}+\frac{\alpha}{2}\left(\kappa_{H B}\right)_{33} B_{0}^{2}-\alpha \frac{\lambda B_{0}}{r}\left(\kappa_{D B}\right)_{13}\right] \Psi=M^{2} \Psi
\end{aligned}
$$

Since the metric is independent of time and symmetrical by translations along the $z$-axis, as well by rotations. It is reasonable to write the solution to Eq. (8) as

$$
\Psi(t, r, \phi, z)=e^{i(-E t+l \phi+k z)} \psi(r)
$$

where $E$ is the energy of the particle, $l=0, \pm 1, \pm 2, \ldots$ are the eigenvalues of the $z$-component of the angular momentum operator, and $k$ is a constant.

Substituting the solution (9) into the Eq. (8), we obtain the following radial wave-equation for $\psi(r)$ :

$$
\psi^{\prime \prime}(r)+\frac{1}{r} \psi^{\prime}(r)+\left(\Lambda-M^{2} \omega^{2} r^{2}-\frac{j^{2}}{r^{2}}-\frac{a}{r}\right) \psi(r)=0,
$$


where

$$
\begin{aligned}
& \Lambda=E^{2}-M^{2}-k^{2}+\frac{1}{2} \alpha B_{0}^{2}\left(\kappa_{H B}\right)_{33}-2 M \omega, \\
& j=\sqrt{l^{2}+\frac{1}{2} \alpha \lambda^{2}\left(\kappa_{D E}\right)_{11}} \\
& a=\alpha \lambda B_{0}\left(\kappa_{D B}\right)_{13} .
\end{aligned}
$$

Let us perform a change of variables given by $x=\sqrt{M \omega} r$. Then, we have

$$
\psi^{\prime \prime}(x)+\frac{1}{x} \psi^{\prime}(x)+\left(\zeta-x^{2}-\frac{j^{2}}{x^{2}}-\frac{\eta}{x}\right) \psi(x)=0,
$$

where

$$
\zeta=\frac{\Lambda}{M \omega} \quad, \quad \eta=\frac{a}{\sqrt{M \omega}} .
$$

By analysing the asymptotic behaviour of Eq. (12) at $x \rightarrow 0$ and $x \rightarrow \infty$, we have a solution to Eq. (12) that can be written in terms of an unknown function $F(x)$ as

$$
\psi(x)=x^{j} e^{-\frac{x^{2}}{2}} H(x)
$$

Thereby, substituting Eq. (14) into the Eq. (12), we obtain

$$
H^{\prime \prime}(x)+\left[\frac{1+2 j}{x}-2 x\right] H^{\prime}(x)+\left[-\frac{\eta}{x}+\Theta\right] H(x)=0,
$$

where $\Theta=\zeta-2(1+j)$.

Equation (15) is the biconfluent Heun's differential equation $[1,2,4,5$, $6,7,8,9,11,37,38,39,40]$ with $H(x)$ is the Heun polynomials function.

The above equation (15) can be solved by the Frobenius method. Writing the solution as a power series expansion around the origin [41]:

$$
H(x)=\sum_{i=0}^{\infty} d_{i} x^{i}
$$

Substituting the power series solution into the Eq. (15), we obtain the following recurrence relation

$$
d_{n+2}=\frac{1}{(n+2)(n+2+2 j)}\left[\eta d_{n+1}-(\Theta-2 n) d_{n}\right]
$$


With few coefficients are

$$
\begin{aligned}
& d_{1}=\frac{\eta}{1+2 j} d_{0} \\
& d_{2}=\frac{1}{4(1+j)}\left[\eta d_{1}-\Theta d_{0}\right] .
\end{aligned}
$$

The power series expansion $H(x)$ becomes a polynomial of degree $n$ by imposing the following two conditions $[1,2,4,5,6,7,8,9,11,37]$

$$
\Theta=0 \quad, \quad d_{n+2}=0 \quad(n=1,2,3, \ldots) .
$$

By analysing the first condition, we have

$E_{n, l}= \pm \sqrt{M^{2}+k^{2}+2 M \omega\left(n+2+\sqrt{l^{2}+\frac{1}{2} \alpha \lambda^{2}\left(\kappa_{D E}\right)_{11}}\right)-\frac{1}{2} \alpha B_{0}^{2}\left(\kappa_{H B}\right)_{33} .}$

Hence, Eq. (20) is the non-compact expression of the energy eigenvalues equation of a relativistic quantum oscillator field under the effects of a Coulomb-type central potential induced by Lorentz symmetry violation background out of the Standard Model Extension. Note that (20) is not the general expression of the energy eigenvalues. One can obtain the individual energy level and the corresponding eigenfunction one by one imposing the recurrence condition $d_{n+1}=0$ on the eigenvalue problem.

The wave-function is given by

$$
\psi(x)=x^{\sqrt{l^{2}+\frac{1}{2} \alpha \lambda^{2}\left(\kappa_{D E}\right)_{11}}} e^{-\frac{x^{2}}{2}} H(x),
$$

where $H(x)$ is the polynomial of degree $n$ contains $(n+1)$ terms in the expansion.

For $\left(\kappa_{D E}\right)_{11}=0$, the energy eigenvalues (20) becomes

$$
E_{n, l}= \pm \sqrt{M^{2}+k^{2}-\frac{1}{2} \alpha B_{0}^{2}\left(\kappa_{H B}\right)_{33}+2 M \omega(n+2+|l|)} .
$$

And the wave-function is given by

$$
\psi(x)=x^{|l|} e^{-\frac{x^{2}}{2}} H(x) .
$$


Again for $\left(\kappa_{H B}\right)_{33}=0$, the energy eigenvalues (20) becomes

$$
E_{n, l}= \pm \sqrt{M^{2}+k^{2}+2 M \omega\left(n+2+\sqrt{l^{2}+\frac{1}{2} \alpha \lambda^{2}\left(\kappa_{D E}\right)_{11}}\right)}
$$

And the wave-function is Eq. (21).

Now, we impose the recurrence condition $d_{n+1}=0$ on the eigenvalue to find the individual energy level and eigenfunction as done in Refs. $[2,4,5$, $6,7,8,9,11,37]$. For the radial mode $n=1$, we have $d_{2}=0$ and $\Theta=2$. Thus from (18), we have

$$
\frac{\eta}{1+2 j} d_{0}=\frac{\eta}{2} d_{0} \Rightarrow \omega_{1, l}=\frac{a^{2}}{2 M(1+2 j)}=\frac{\left(\alpha \lambda B_{0}\left(\kappa_{D B}\right)_{13}\right)^{2}}{2 M(1+2 j)}
$$

a constraint on the oscillator frequency $\omega$ which gives us that, by choosing the oscillator frequency as a parameter that can be adjusted, hence, some specific values of the oscillator frequency are permitted in order to achieve a first degree polynomial solution to the function $H(r)$. We have obtained Eq. (25) which corresponds to the possible values of the oscillator frequency associated with the lowest state of the system defined by the radial mode $n=1$, and thus, satisfy the asymptotic behaviour of the radial wave-function when $x \rightarrow$ 0 and $x \rightarrow \infty$. Furthermore, the possible values of the oscillator frequency depend on the parameters that establish the scenario of Lorentz symmetry violation $\left(g, \lambda, B_{0},\left(\kappa_{D B}\right)_{13}\right)$ and the quantum number of the system $\{n, l\}$.

Thus, the lowest state energy level for the radial mode $n=1$ using (16) becomes

$E_{1, l}= \pm \sqrt{M^{2}+k^{2}-\frac{1}{2} \alpha B_{0}^{2}\left(\kappa_{H B}\right)_{33}+2 M \omega_{1, l}\left(3+\sqrt{l^{2}+\frac{1}{2} \alpha \lambda^{2}\left(\kappa_{D E}\right)_{11}}\right)}$

The ground state wave-function is

$$
\psi_{1, l}(x)=x^{\sqrt{l^{2}+\frac{1}{2} \alpha \lambda^{2}\left(\kappa_{D E}\right)_{11}}} e^{-\frac{x^{2}}{2}}\left(1+d_{1} x\right),
$$


where we have chosen $d_{0}=1$ and

$$
d_{1}=\frac{1}{\sqrt{\frac{1}{2}+\sqrt{l^{2}+\frac{1}{2} \alpha \lambda^{2}\left(\kappa_{D E}\right)_{11}}}} .
$$

We have established the background of the Lorentz symmetry violation defined by a tensor that governs the Lorentz symmetry breaking possessing the non-null components $\left(\kappa_{D B}\right)_{13}=$ const, $\left(\kappa_{D E}\right)_{11}=$ const and $\left(\kappa_{H B}\right)_{33}=$ const, Coulomb-type radial electric field produced by electric charge distribution, and a uniform magnetic along the $z$-direction. We have seen that the presence of the Lorentz symmetry breaking parameters modified the lowest state energy level and the ground state wave-function for each radial mode defined by $n=1,2,3, \ldots$ in comparison to the Landau levels.

\section{Special case $\left(\kappa_{D B}\right)_{13}=0$}

Here we have choose a Lorentz symmetry parameter $\left(\kappa_{D B}\right)_{13}=0$ and others are non-null in the same relativistic quantum system. Therefore, the radial wave-equation becomes

$$
\psi^{\prime \prime}(r)+\frac{1}{r} \psi^{\prime}(r)+\left(\Lambda-M^{2} \omega^{2} r^{2}-\frac{j^{2}}{r^{2}}\right) \psi(r)=0,
$$

Performing a change of variable $x=M \omega r^{2}$ into the equation (29), we have

$$
\psi^{\prime \prime}(x)+\frac{1}{x} \psi^{\prime}(x)+\frac{1}{4 x}\left(\frac{\Lambda}{M \omega}-x-\frac{j^{2}}{x}\right) \psi(x)=0,
$$

The above equation can be transform to the following differential equation $[42]$

$$
\psi^{\prime \prime}(x)+\frac{1}{x} \psi^{\prime}(x)+\frac{1}{x^{2}}\left(-\xi_{1} x^{2}+\xi_{2} x-\xi_{3}\right) \psi(x)=0
$$

where

$$
\xi_{1}=\frac{1}{4} \quad, \quad \xi_{2}=\frac{\Lambda}{4 M \omega} \quad, \quad \xi_{3}=\frac{j^{2}}{4} .
$$


Comparing Eq. (31) with Eq. (A.1) in Ref. [43]

$$
\begin{aligned}
& \alpha_{1}=1 \quad, \quad \alpha_{2}=0 \quad, \quad \alpha_{3}=0 \quad, \quad \alpha_{4}=0 \quad, \quad \alpha_{5}=0, \\
& \alpha_{6}=\xi_{1} \quad, \quad \alpha_{7}=-\xi_{2} \quad, \quad \alpha_{8}=\xi_{3} \quad, \quad \alpha_{9}=\xi_{1} \quad, \quad \alpha_{10}=1+2 \sqrt{\xi_{3}}, \\
& \alpha_{11}=2 \sqrt{\xi_{1}} \quad, \quad \alpha_{12}=\sqrt{\xi_{3}} \quad, \quad \alpha_{13}=-\sqrt{\xi_{1}} .
\end{aligned}
$$

The eigenvalues equation using Eqs. (32)-(33) into the Eq. (A.8) in Ref. [43]

$$
E_{m, l}= \pm \sqrt{M^{2}+k^{2}+2 M \omega(2 m+1+j)-\frac{1}{2} \alpha B_{0}\left(\kappa_{H B}\right)_{33}}
$$

where $m=0,1,2, \ldots$ and $j=\sqrt{l^{2}+\frac{1}{2} \alpha \lambda^{2}\left(\kappa_{D E}\right)_{11}}$.

We can see that the presence of the tensor parameter $\left(\kappa_{D E}\right)_{11}$ and $\left(\kappa_{H B}\right)_{13}$ modified the energy spectrum and the wave-function of a scalar particle.

The normalized radial wave-function is given by

$$
\psi_{m, l}(x)=|N|_{m, l} x^{\frac{j}{2}} e^{-\frac{x}{2}} L_{m}^{(j)}(x),
$$

where $|N|_{m, l}=\left(\frac{m !}{(m+j) !}\right)^{\frac{1}{2}}$ is the normalization constant and $L_{m}^{(j)}(x)$ is the generalized Laguerre polynomials and are orthogonal over $(0, \infty]$ with respect to the measure with weighting function $x^{j} e^{-x}$ as

$$
\int_{0}^{\infty} x^{j} e^{-x} L_{m}^{(j)}(x) L_{m^{\prime}}^{(j)}(x) d x=\left(\frac{(m+j) !}{m !}\right) \delta_{m m^{\prime}}
$$

For zero Lorentz symmetry parameter $\left(\kappa_{H B}\right)_{33}=0$, the energy eigenvalues (34) becomes

$$
E_{m, l}= \pm \sqrt{M^{2}+k^{2}+2 M \omega\left(2 m+1+\sqrt{l^{2}+\frac{1}{2} \alpha \lambda^{2}\left(\kappa_{D E}\right)_{11}}\right)}
$$

with the same normalized radial wave-function (35). Still we can see that the parameter $\left(\kappa_{D E}\right)_{11}$ modified the energy spectrum and the wave-function of a scalar particle. 
Again for $\left(\kappa_{H B}\right)_{33}=0$ and $\left(\kappa_{D E}\right)_{13}=0$, the energy eigenvalue for the Landau levels using (34) becomes

$$
E_{m, l}= \pm \sqrt{M^{2}+k^{2}+2 M \omega(2 m+1+|l|)}
$$

The normalized radial wave-function is given by

$$
\psi_{m, l}(x)=|N|_{m, l} x^{\frac{|l|}{2}} e^{-\frac{x}{2}} L_{m}^{(|l|)}(x),
$$

where $|N|_{m, l}=\left(\frac{m !}{(m+|l|) !}\right)^{\frac{1}{2}}$ is the normalization constant and $L_{m}^{(|l|)}(x)$ is the generalized Laguerre polynomials and are orthogonal over $(0, \infty]$ with respect to the measure with weighting function $x^{|l|} e^{-x}$ as

$$
\int_{0}^{\infty} x^{|l|} e^{-x} L_{m}^{(|l|)}(x) L_{m^{\prime}}^{(|l|)}(x) d x=\left(\frac{(m+|l|) !}{m !}\right) \delta_{m m^{\prime}}
$$

Thus we can see that the energy eigenvalues (37) and or (34) get modified by the parameter of the tensor $\left(\kappa_{F}\right)_{\mu \nu \alpha \beta}$ that governs the Lorentz symmetry breaking effects in comparison the Landau levels.

\section{Conclusions}

We have investigated a scalar particle in the background of central potential induced by Lorentz symmetry breaking effects. In our studies, we have relaxed the renormalization property because of tiny values contribute by the terms that violate Lorentz symmetry whose energy scales goes beyond the Standard Model. We have shown that bound states solutions to the KleinGordon oscillator can be obtained in the Lorentz symmetry violation scenario defined by an electric field proportional to inverse of radial distance and a constant magnetic field, and the tensor $\left(\kappa_{F}\right)_{\mu \nu \alpha \beta}$ that governs the Lorentz symmetry breaking effects having non-null components $\left(\kappa_{D B}\right)_{13}=$ const, $\left(\kappa_{D E}\right)_{11}=$ const and $\left(\kappa_{H B}\right)_{33}=$ const. We derive the radial wave-equation of the KG-oscillator equation and choosing a suitable function the biconfluent Heun differential form is arrived. Finally, using the power series method 
and truncating the power series solution, we have obtained the non-compact expression of the energy eigenvalues Eq. (20) and the wave-function Eq. (21). We have seen that the presence of Lorentz symmetry breaking terms modified the energy spectrum in comparison to the Landau levels. Further, by imposing the additional recurrence condition $d_{n+1}=0$ on the eigenvalue problem, one can obtain the individual energy level and the wave-function, for an example, the lowest state energy level Eq. (26) and the corresponding wave-function Eqs. (27)-(28) for the radial mode $n=1$ instead of $n=0$. This effect arises due to the presence of Lorentz symmetry breaking parameters. In this analysis, we have seen that the oscillator frequency $\omega$ depends on the quantum numbers $\{n, l\}$ of the system and the parameters that establish the scenario of Lorentz symmetry violation $\left(g, \lambda, B_{0},\left(\kappa_{D B}\right)_{13}\right)$, for example, Eq. (25) is the possible values of the oscillator frequency for the radial mode $n=1$. Following the similar technique, one can obtain relations for the oscillator frequency $\omega_{2, l}$ for the radial mode $n=2$ and so on. Thus the dependence of the angular frequency of the oscillator $\omega$ on the quantum numbers of the system shows a quantum effect.

Furthermore, we have discussed a very special case corresponds one of the Lorentz symmetry term $\left(\kappa_{D B}\right)_{13}=0$ in the considered quantum system and other are non-zero. We have derived the radial wave-equation of the KGequation and finally using the Nikiforov-Uvarov method, we have obtained the energy eigenvalues (34) and the normalized eigenfunction (35). We have seen that the presence of the non-null components $\left(\kappa_{D E}\right)_{11}=$ const and $\left(\kappa_{H B}\right)_{33}$ of the tensor that governs the Lorentz symmetry breaking effects modified the energy spectrum and the wave-function obtained in the both cases.

\section{Data Availability}

No data has been used to prepare this manuscript. 


\section{Conflict of Interest}

Author declares that there is no conflict of interest regarding publication this paper.

\section{References}

[1] F. Ahmed, Eur. Phys. J. C 78, 598 (2018).

[2] F. Ahmed, Adv. High Energy Phys. 2020, 5691025 (2020).

[3] A. Boumali and N. Messai, Can. J. Phys. 92, 1460 (2014).

[4] J. Carvalho, A. M. M. Carvalho, E. Cavalcante, and C. Furtado, Eur. Phys. J. C 76, 365 (2016).

[5] Z. Wang, Z. Long, C. Long and M. Wu, Eur Phys. J. Plus 130, 36 (2015).

[6] M. Hosseinpour, H. Hassanabadi and Marc de Montigny, Int. J. Geom. Meths Mod. Phys. 15, 1850165 (2018).

[7] F. Ahmed, Int. J. Geom. Meths Mod. Phys. 17, 2050138 (2020).

[8] B. -Q. Wang, Z. -W. Long, C. -Y. Long and S. -R. Wu, Mod. Phys. Lett. A 33, 1850025 (2018).

[9] M. Hosseini, H. Hassanabadi, S. Hassanabadi and P. Sedaghatnia, Int. J. Geom. Meths. Mod. Phys. 16, 1950054 (2019).

[10] H. Sobhani, H. Hassanabadi and W. S. Chung, Int. J. Geom. Meths Mod. Phys. 15, 1850037 (2018).

[11] F. Ahmed, EPL 130, 40003 (2020).

[12] S. Bruce and P. Minning, II Nuovo Cimento A 106, 711 (1993). 
[13] M. Moshinsky and A. Szczepaniak, J. Phys. A : Math. Gen. 22, L817 (1989).

[14] D. Colladay and V. A. Kostelecky, Phys. Rev. D 55, 6760 (1997).

[15] D. Colladay and V. A. Kostelecky, Phys. Rev. D 58, 116002 (1998).

[16] V. A. Kostelecky and S. Samuel, Phys. Rev. Lett. 63, 224 (1989).

[17] V. A. Kostelecky and S. Samuel, Phys. Rev. Lett. 66, 1811 (1991).

[18] V. A. Kostelecky and S. Samuel, Phys. Rev. D 39, 683 (1989).

[19] V. A. Kostelecky and S. Samuel, Phys. Rev. D 40, 1886 (1989).

[20] V. A. Kostelecky and R. Potting, Nucl. Phys. B 359, 545 (1991).

[21] V. A. Kostelecky and R. Potting, Phys. Lett. B 381, 89 (1996).

[22] V. A. Kostelecky and R. Potting, Phys. Rev. D 51, 3923 (1995).

[23] V. A. Kostelecky and R. Lehnert, Phys. Rev. D 63, 065008 (2001).

[24] S. Carroll, G. Field and R. Jackiw, Phys. Rev. D 41, 1231 (1990).

[25] A. P. Baeta Scarpelli, H. Belich, J. L. Boldo, L. P. Colatto, J. A. HelayelNeto, A. L. M. A. Nogueira, Nucl. Phys. B (Proc. Suppl.) 127, 105 (2004).

[26] V. A. Kostelecky and M. Mewes, Phys. Rev. Lett. 87, 251304 (2001).

[27] V. A. Kostelecky and M. Mewes, Phys. Rev. D 66, 056005 (2002).

[28] H. Belich, F. J. L. Leal, H. L. C. Louzada and M. T. D. Orlando, Phys. Rev. D 86, 125037 (2012).

[29] K. Bakke and H. Belich, Ann. Phys. (N. Y.) 360, 596 (2015). 
[30] R. L. L. Vitoria, H. Belich and K. Bakke, Eur. Phys. J. Plus 132, 25 (2017).

[31] K. Bakke and H. Belich, Ann. Phys. (N. Y.) 354, 1 (2015).

[32] K. Bakke and H. Belich, Ann. Phys. (N. Y.) 373, 115 (2016).

[33] R. L. L. Vitoria and H. Belich, Eur. Phys. J. C 78, 999 (2018).

[34] F. Ahmed, IJMPA (2021), DOI:10.1142/S0217751X21501281.

[35] R. L. L. Vitoria, H. Belich and K. Bakke, Adv. High Energy Phys. 2017, 6893084 (2017).

[36] R. L. L. Vitória, K. Bakke and H. Belich, Ann. Phys. (N. Y.) 399, 117 (2018).

[37] E. R. Figueiredo Medeiros, E. R. Bezerra de Mello, Eur. Phys. J. C 72, 2051 (2012).

[38] A. Ronveaux, Heun's Differential Equations, Oxford University Press, Oxford ( 1995).

[39] S. Y. Slavyanov and W. Lay, Special Functions: A Unified Theory Based in Singularities, Oxford University Press, New York (2000).

[40] M. Abramowitz and I. A. Stegum, Hand book of Mathematical Functions, Dover Publications Inc., New York (1965).

[41] G. B. Arfken and H. J. Weber, Mathematical Methods For Physicists, Elsevier Academic Press, London (2005).

[42] A. F. Nikiforov and V. B. Uvarov, Special functions of mathematical physics (Birkhauser, Basel, 1988).

[43] F. Ahmed, Eur. Phys. J. C 79, 682 (2019). 